J. Appl. Cryst. (1974). 7, 190

Measurements of Radius of Gyration and Persistence Length in Bulk Atactic Polystyrene by Low-Angle Neutron Scattering

\author{
By G. D. Wignall \\ Imperial Chemical Industries Corporate Laboratory, Runcorn, Cheshire, England, \\ J. SCHELTEN \\ Institut für Festkörperforschung der Kernforschungsanlage Jülich, Germany (BRD) \\ AND D. G. H. BALlaRd \\ Imperial Chemical Industries Corporate Laboratory, Runcorn, Cheshire, England
}

\begin{abstract}
The radius of gyration, $r_{g}$, of atactic polystyrene molecules in the bulk polymer has been measured by low-angle neutron scattering from dilute solid solutions of poly-proto-styrene in poly-deutero-styrene with concentrations of $5 \%$ and $0.5 \%$ tagged solute molecules. The measured molecular dimensions agree within the experimental errors $( \pm 5 \%)$ with the unperturbed dimensions of the polymer chain as determined from the solution properties of polymer. The data in the high- $K$ region $[K=4 \pi / \lambda) \sin \theta]$ of the scattering curve is interpreted according to the theory due to O. Kratky [Colloid. Z. 182, 7-24] and leads to an estimate of the persistence length $\sim 10 \AA$. Together with the previous result, this indicates that the molecular configuration corresponds to a random flight chain, similar to the configuration in a $\theta$ solvent. This confirm the prediction of P. J. Flory [J. Chem. Phys. (1949). 17, 303-310], and rules out other model configurations for this polymer. Measurements of $r_{g}$ throughout the glass transition region are important for understanding this transition in organic polymers, and measurements on polystyrene in the region $20<T<160^{\circ} \mathrm{C}$ are reviewed. $r_{g}$ is essentially independent of temperature in this region, and this behaviour is consistent with the existence of an unperturbed random flight chain, both above and below the glass transition region.
\end{abstract}

J. Appl. Cryst. (1974). 7, 190

\title{
Determination of Absolute Values of the Density Fluctuation in Amorphous and Semicrystalline Polymers
}

\author{
By J. Rathue And W. Ruland \\ Universität Marburg, Fachbereich Physikalische Chemie, Bereich Polymere, Lahnberge, Gebäude H, \\ Germany (BRD)
}

The small-angle scattering of polymers contains a component which is due to the statistical fluctuation of the density produced by imperfections in the long-range or short-range order. This fluctuation is entirely due to thermal motion and is related to the compressibility in the case of a one-component fluid system in thermodynamic equilibrium. In non-equilibrium one-component systems in the solid state, the statistical fluctuations of the density are, at least partly, due to frozen-in disordered structures. In both the equilibrium and the non-equilibrium state, the fluctuation is primarily an overall disorder parameter, the absolute value of which can be used for a quantitative characterization of the state of order. The relationship between compressibility and density fluctuations has been applied for normalizing the scattering curves using liquids of known compressibility. Care was taken in eliminating the effect of multiple scattering in varying the thickness of the samples and extrapolating to zero thickness. The values obtained for semicrystalline polymers vary approximately linearly with crystallinity and can be evaluated using constant values for the amorphous and the crystalline state at a given temperature. For polyethylene at room temperature a difference of at least one order of magnitude has been observed between the density fluctuations of the amorphous and the crystalline state. The absolute values of the density fluctuation can be used to check disorder models. An example is given in terms of a simple disorder model assuming statistical distributions of vacancies in an ordered matrix. The result is at least semi-quantitatively in agreement with results of thermodynamical calculations. In the case of chemically cross-linked polymers the density fluctuation contains a component due to the average number of excess (or deficit) electrons per cross link which can be separated from the density fluctuation of the matrix in studying the total fluctuation as a function of the concentration of cross links. For a rubber cross-linked with dicumylperoxide, a linear relationship between the total density fluctuation and the average number of electrons in a chain between two cross links has been observed from which the density fluctuation of the uncross-linked rubber and the excess electrons per cross link have been determined. The study of the temperature dependence of the density fluctuations in amorphous and semicrystalline polymers offers an interesting possibility to obtain information on the changes in the mobility of the chains and in the perfection of their spatial arrangements. 